\title{
Post-operative outcome following ileal pouch-anal anastomosis (IPAA)
}

\author{
W.P.L. Weerakkody, M.T.D. Silva, D.N. Samarasekera \\ Department of Surgery, Faculty of Medicine, University of Colombo, Sri Lanka
}

Key words: Post-operative; ileal pouch; anal; anastomosis

\begin{abstract}
\section{Introduction}

The ileal pouch-anal anastomosis (IPPA) procedure is the surgery of choice in severe ulcerative colitis (UC) and familial adenomatous polyposis (FAP). The objectives of the study were to assess symptomatic improvement, complications, effect on sexual function and quality of life (QOL) following IPAA procedure.
\end{abstract}

\section{Methods}

Twenty one patients who underwent IPAA procedure at a tertiary care unit with a minimum of one year follow-up from 2009, were included. QOL was assessed retrospectively prior to surgery and one year after surgery using Cleveland Global Quality of Life Score (CGQLS). Details of pre and post-op symptoms, complications and sexual function were obtained using clinical records, operative notes and interviewer administered questionnaire.

\section{Results}

Male: female ratio was 1.33:1. Fourteen (66.7\%) were sexually active and seven (33.3\%) were not. Mean age was 40.48(range, 17-69) years. 13(61.9\%) had UC and 8(38.1\%) had FAP. Mean pre-op CGQL scores for UC and FAP were 0.5754 and 0.7537 respectively. Post-op CGQLS for UC and FAP were 0.7532 and 0.7070 . There was no statistically significant difference between CGQL scores against the indication for surgery $(\mathrm{P}=0.779)$. Stool frequency was significantly reduced in $\mathrm{UC}$ ( $\mathrm{pre}-\mathrm{op}=12.4$, post-op $=5.2$ per day, $\mathrm{p}=0.004$ ) but no difference in FAP (pre-op=2.6, post$o p=3.5$ per day, $p=0.292$ ). Post-operatively, one patient developed erectile dysfunction and another patient developed dyspareunia.

\section{Conclusion}

Correspondence: Nandadeva Samarasekera
E-mail: samarasekera58@yahoo.co.uk
Received: 18-10-2017 Accepted: 18-12-2017
(i) http://orcid.org/0000-0002-7812-9811
DOI: http://doi.org/10.4038/sljs.v35i4.8432

The Sri Lanka Journal of Surgery 2017; 35(4): 8-11
IThere is no significant difference of CGQL scores following IPAA procedure in relation to the surgical indication. IPAA significantly reduced stool frequency per day in UC but not in FAP. Erectile dysfunction and dyspareunia are possible sexual dysfunctions following IPAA procedure

\section{Introduction}

Ulcerative colitis (UC) is a chronic inflammatory bowel disease which involves colon and rectum. Familial adenomatous polyposis (FAP) is an autosomal dominantly inherited disease which leads to adenomatous polyps in large numbers throughout the colon. Both conditions are associated with colorectal cancer whereas, the latter has 100 percent risk of cancer if left untreated.

Ileal pouch-anal anastomosis procedure (IPAA) is the surgery of choice in UC and FAP. In 1978 Parks et al [1] reported four cases undergoing IPAA successfully. Advantages of IPAA include, avoiding a stoma, cosmetically acceptable for the patient and mimics the natural bowel function. Improving quality of life following surgery for UC and FAP is a major part of the management.

\section{Objectives}

- To determine the symptomatic improvement following IPAA

- Describe the pattern of complications following IPAA

- Assess the impact of complications on quality of life

- To study the effect on sexual function following IPAA

- To study the difference of quality of life (QOL) according to the indication for the ileal pouch-anal anastomosis procedure

\section{Methods}

Cross sectional descriptive and analytical retrospective study design was adopted. The study was conducted in the Professorial Surgical Unit, National Hospital of Sri Lanka (NHSL) on patients who underwent IPAA procedure from April 2009 to May 2016. Patients who underwent IPAA, with a minimum follow up period of one year were included in the study. Patients with psychiatric conditions and other conditions which can affect the quality of life considerably (i.e. malignancies, diabetes mellitus, osteoarthritis, 
rheumatoid arthritis etc.) were excluded from the study. Ethics clearance was obtained from ethics review committee of the National Hospital Sri Lanka. Informed written consent from patients to participate in the research was obtained prior to the interview.

Interviewer administered questionnaire was administered according to their preferable language. The interviewers were uniformly trained regarding the administration of the questionnaire. Basic socioeconomic details were obtained from the patients. Additionally, clinic records and operative notes of the participants were reviewed. Sexual dysfunction was assessed by taking a detailed sexual history in a privacy secured clinic room comparing sexual function preoperatively and one year after surgery.

Pre and post-operative symptoms were assessed using clinical history, clinic records and operative notes. Stool frequency per day, per rectal bleeding, loss of appetite and extra gastro intestinal symptoms like fever, joint pain, red eye, back pain with immobility were assessed pre-operatively and one year after IPAA.

Cleveland Global Quality of Life Score (CGQLS) validated in 1999 by Fazio et al [2] was used to assess the quality of life following Ileal pouch-anal anastomosis procedure. CGQLS consists of 3 components. They are current quality of life, current quality of health and current energy respectively. Each component is given marks out of 10 and average CGQL score is calculated.

The enrolled patients were educated on the CGQLS assessment tool. Subsequently, they were asked to mark the three components of CGQL score in two separate scales recalling the quality of life prior to surgery and one year after surgery.

The questionnaire assessed the details given below.

a) Gender and socioeconomic details

b) Year and the indication of the surgery

c) Cleveland Global Quality of Life assessment tool

d) Symptom assessment

e) Post-operative complications assessment

f) Assessment of the sexual function

\section{Results}

Twenty one patients were incorporated into the study. Male: Female ratio was $1.33: 1$. Thirteen patients $(61.9 \%)$ had UC and eight (38.1\%) had FAP. Mean age was 40.48 (range, 17 to 69) years.

Mean pre-operative CGQL score of all 21 patients was 0.6433 . One year after surgery, mean CGQL score was 0.7365 .

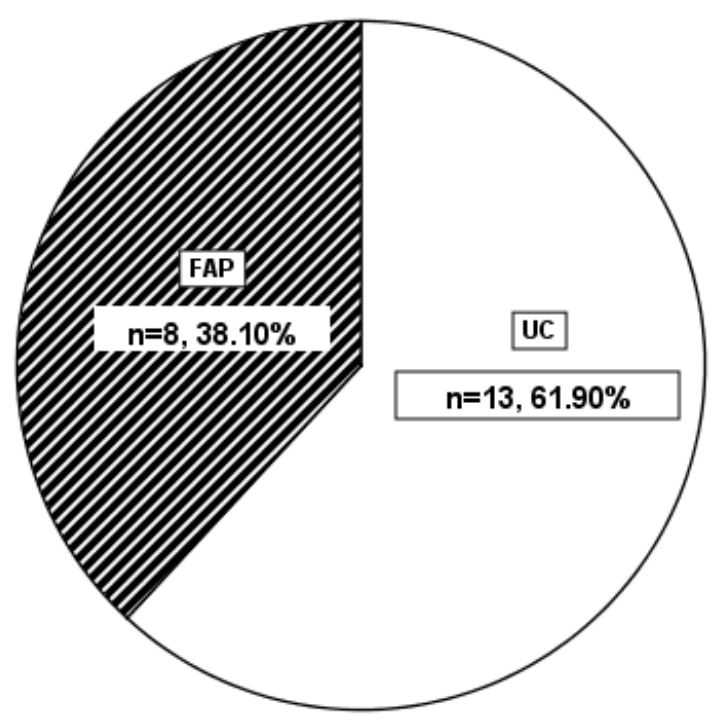

Figure 1. Indication for surgery

\section{Statistical analysis}

Post-operative CGQL scores were compared against the indication for surgery after controlling the effect of preoperative CGQL score using analysis of covariance (ANCOVA) test. There was no statistically significant effect of surgical indication on post-operative CGQL scores after controlling for the effect of pre-operative CGQL scores $(\mathrm{p}=0.779)$.

Commonest pre-operative symptom encountered was per rectal bleeding which was present in $20(95.2 \%)$ patients. Twelve $(57.1 \%)$ patients had loss of appetite prior to surgery. Twelve $(92.3 \%)$ out of 13 patients with UC had extra gastrointestinal symptoms pre-operatively. Arthralgia was present in $9(69.2 \%)$ patients. $7(53.8 \%)$ had fever preoperatively. Red eye suggestive of uveitis and severe back pain with reduced mobility suggestive of ankylosing spondylitis were found in $2(15.4 \%)$ patients each.

Symptoms were assessed one year after surgery. Per rectal bleeding persisted in $2(9.5 \%)$ patients, one from the UC group and the other from the FAP group. 3 (14.3\%) patients had loss of appetite one year after surgery. Five (38.4\%) out of 13 patients with UC had extra gastrointestinal symptoms one year following IPAA. Arthralgia was present in $3(23.1 \%)$ patients one year after surgery. No patients had fever after one year of surgery. Red eye suggestive of uveitis was not found. Severe back pain with reduced mobility suggestive of ankylosing spondylitis was found in $2(15.4 \%)$ patients.

Table 1. Symptom assessment

\begin{tabular}{|l|c|c|}
\hline & Pre-operative & $\begin{array}{c}\text { One year following } \\
\text { IPAA }\end{array}$ \\
\hline Per rectal bleeding & $20(95.2 \%)$ & $2(9.5 \%)$ \\
\hline Loss of appetite & $12(57.1 \%)$ & $3(14.3 \%)$ \\
\hline
\end{tabular}

The Sri Lanka Journal of Surgery 2017; 35(4): 8-11 
Table 2. Extra gastrointestinal symptom assessment

\begin{tabular}{|l|c|c|}
\hline & Pre-operative & $\begin{array}{c}\text { One year } \\
\text { following IPAA }\end{array}$ \\
\hline $\begin{array}{l}\text { Extra gastrointestinal } \\
\text { symptoms }\end{array}$ & $12(92.3 \%)$ & $5(38.4 \%)$ \\
\hline Arthralgia & $9(69.2 \%)$ & $3(23.1 \%)$ \\
\hline Fever & $7(53.8 \%)$ & 0 \\
\hline Red eye (Uveitis) & $2(15.4 \%)$ & 0 \\
\hline $\begin{array}{l}\text { Severe back pain } \\
\text { (Ankylosing spondylitis) }\end{array}$ & $2(15.4 \%)$ & $2(15.4 \%)$ \\
\hline
\end{tabular}

Mean pre-operative stool frequency per day was 12.4 in the UC group. One year after surgery, it reduced to 5.2 per day one year after surgery. In FAP group, mean pre-operative stool frequency per day was 2.6. One year after surgery, it had increased to 3.5 per day.

Wilcoxon signed rank test was used to analyse the preoperative and one year post-operative mean stool frequencies per day in UC and FAP groups separately.

Among patients with UC, there was a statistically significant reduction in post-operative stool frequency per day compared to pre-operative stool frequency per day $(\mathrm{p}=0.004)$.

Among patients with FAP, there was no statistically significant difference in post-operative stool frequency per day compared to pre-operative stool frequency per day $(\mathrm{p}=0.292)$.

Table 3. Complications

\begin{tabular}{|l|c|c|}
\hline & $\begin{array}{c}\text { Ulcerative } \\
\text { colitis }\end{array}$ & $\begin{array}{c}\text { Familial adenomatous } \\
\text { polyposis }\end{array}$ \\
\hline $\begin{array}{l}\text { Abdominal } \\
\text { wound infection }\end{array}$ & $4(19 \%)$ & $5(23.8 \%)$ \\
\hline Anaemia & $4(19 \%)$ & $3(14.3)$ \\
\hline Pelvic abscess & $1(4.8 \%)$ & $1(4.8 \%)$ \\
\hline Incisional hernia & $1(4.8 \%)$ & $1(4.8 \%)$ \\
\hline
\end{tabular}

All the patients were reviewed for complications within one year of surgery. Commonest complication was abdominal wound infection which was present in $9(42.9 \%)$ patients.

Out of 9 those patients who had abdominal wound infections, 4 were in UC group and 5 were in FAP group. Anaemia was found in $7(33.3 \%)$ patients after one year of surgery. Out of 7 anaemic patients, 4 were from UC group and 3 were from the FAP group. Two patients (9.5\%) developed pelvic abscesses. They were one each from UC and the FAP groups. Two patients $(9.5 \%)$ developed incisional hernia. They were one each from UC and the FAP groups.
$14(66.7 \%)$ patients were sexually active pre-operatively. Out of 14 sexually active patients, $10(47.6 \%)$ were from the UC group and $4(19 \%)$ were from the FAP group. Postoperatively, there was no change in the number of sexually active patients.

One patient in the UC group who had normal sexual function pre-operatively developed complete erectile dysfunction after IPAA.

Table 4. Sexual function

\begin{tabular}{|l|c|c|}
\hline & $\begin{array}{c}\text { Ulcerative } \\
\text { colitis }\end{array}$ & $\begin{array}{c}\text { Familial adenomatous } \\
\text { polyposis }\end{array}$ \\
\hline $\begin{array}{l}\text { Pre-operatively } \\
\text { sexually active }\end{array}$ & $10(47.6 \%)$ & $4(19 \%)$ \\
\hline $\begin{array}{l}\text { Post-operatively } \\
\text { sexually active }\end{array}$ & $10(47.6 \%)$ & $4(19 \%)$ \\
\hline $\begin{array}{l}\text { Erectile } \\
\text { dysfunction }\end{array}$ & $1(4.8 \%)$ & 0 \\
\hline Dyspareunia & 0 & $1(4.8 \%)$ \\
\hline
\end{tabular}

Impact of complications on post-operative CGQLS was analysed.

Table 5. Impact of complications on CGQLS

\begin{tabular}{|l|l|l|}
\hline & $\begin{array}{l}\text { Pre-op } \\
\text { CGQLS Mean }\end{array}$ & $\begin{array}{l}\text { Post-op } \\
\text { CGQLS Mean }\end{array}$ \\
\hline $\begin{array}{l}\text { Abdominal } \\
\text { wound infections }\end{array}$ & 0.6383 & 0.6593 \\
\hline Anaemia & 0.6286 & 0.7366 \\
\hline Pelvic Abscess & 0.5650 & 0.7300 \\
\hline Incisional hernia & 0.5500 & 0.7000 \\
\hline
\end{tabular}

One female patient in the FAP group who had normal sexual function pre-operatively developed superficial dyspareunia after surgery.

Post-op CGQL scores were compared using repeated ANOVA. The differences between the means of post-op CGQL scores of main complications encountered were not statistically significant $(\mathrm{p}>0.05)$.

\section{Discussion}

Ileal pouch-anal anastomosis procedure (IPAA) is the surgery of choice in UC and FAP. In UC, it is carried out when the symptoms are severe and cannot be controlled with medical treatment. But in FAP, it is carried out to prevent the occurrence of malignancy which is inevitable if left untreated. FAP patients are usually detected on screening and they are relatively asymptomatic compared to UC patients.

In 1989, Pemberton et al [3] in a controlled trial compared 298 patients who underwent IPAA with 406 patients with Brooke ileostomy and concluded that after IPAA, patients experienced significant advantages in performing daily activities compared to patients with Brooke ileostomy.

Duminda et al [4] in 2010 in a prospective study concluded 
that IPAA increases the CGQL scores in UC group significantly after 1 year. But no significant difference was found in FAP group. Our study was designed to compare CGQL scores against the surgical indication to assess the symptomatic improvement and to describe the occurrence of sexual dysfunction and other complications. When comparing the CGQL scores after one year of surgery against the indication, influence of pre-operative CGQL scores has to be controlled since quality of life of UC group is compromised due to presence of symptoms despite the medical treatment. Compared to a previous study [4], this study showed the same trend of mean CGQL scores preoperatively and post-operatively after one year. After controlling the influence of pre-operative CGQL scores, no significant difference of post-operative CGQL scores after one year of surgery was found implying that IPAA procedure is acceptable in both UC and FAP.

In assessment of daily stool frequency, significant postoperative reduction was found in UC patients. Despite a major surgical procedure carried out in relatively normal FAP patients, there is no statistically significant rise in daily stool frequency within one year of surgery. Both the above findings favour ileal pouch-anal anastomosis. IPAA has also reduced the number of patients who suffered from gastrointestinal and extra gastrointestinal symptoms after the surgery.

Septic complications were commonly found despite the indication for surgery. Anaemia was another commonly encountered complication which may be preventable. The analysis of impact of complications on CGQL scores showed that despite the complications the IPAA procedure improved the quality of life.

Metcalf et al [5] in 1986 and Damgaard et al [6] in 1995 reported improvement of sexual function following IPAA procedure. In our study, two patients who had normal sexual function pre-operatively developed sexual dysfunctions after surgery. It implies that IPAA can be associated with sexual dysfunction which can be explained by damage to the pelvic nerves during surgery.
Main limitation of the study was the limited number of subjects. This study was done retrospectively and therefore recall bias played a role in obtaining information from the patients.

\section{Conclusion}

This study concludes that there is no significant difference of CGQL scores following IPAA procedure in relation to the surgical indication. It also showed that, there is significant reduction of stool frequency per day post-operatively in UC but no difference in FAP. Pouch related complications made no significant difference on post-op CGQL scores. Erectile dysfunction and dyspareunia are possible sexual dysfunctions following IPAA procedure.

All authors disclose no conflict of interest. The study was conducted in accordance with the ethical standards of the relevant institutional or national ethics committee and the Helsinki Declaration of 1975, as revised in 2000 .

\section{References}

1. Parks AG, Nicholls RJ. Proctocolectomy without ileostomy for ulcerative colitis. British Medical Journal 1978; 2: 85-8. https://doi.org/10.1136/bmj.2.6130.85

2. Fazio VW, O'Riordain MG, Lavery IC, et al. Long-term functional outcome and quality of life after stapled restorative proctocolectomy. Annals of Surgery 1999; 230: 575-86 https://doi.org/10.1097/00000658-199910000-00013

3. Pemberton JH, Phillips SF, Ready RR, Zinsmeister AR, Bearhs $\mathrm{OH}$. Quality of life after Brooke ileostomy and ileal pouch-anal anastomosis: comparison of performance status.Annals of Surgery 1989; 209: 620-8. https://doi.org/10.1097/00000658-198905000-00015

4. Subasinghe D, Samarasekera DN. Quality of life following restorative proctocolectomy for ulcerative colitis and familial adenomatous polyposis. Ceylon Medical Journal 2010; 55: 93 https://doi.org/10.4038/cmj.v55i3.2296

5. Metcalf AM, Dozois RR, Kelly KA, Sexual function in women after proctocolectomy, Annals of surgery 1986; 204(6) : 624-7 https://doi.org/10.1097/00000658-198612000-00002

6. B. Damgaard A, Wettergren P, Kirkegaard, Social and sexual function following ileal pouch-anal anastomosis, Dis Colon Rectum 1995; 38(3): 286-289 https://doi.org/10.1007/BF02055604 